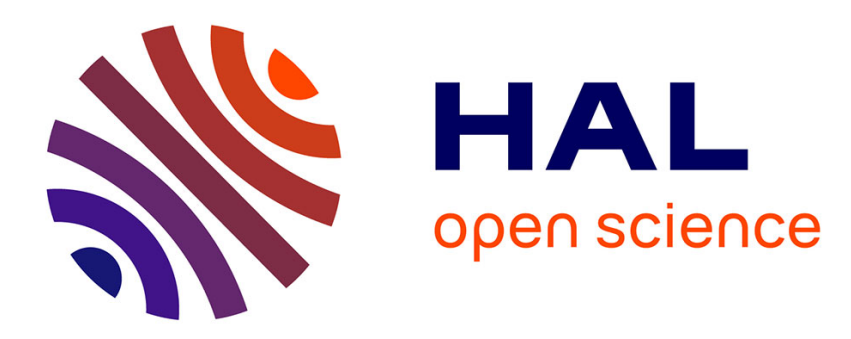

\title{
Energy velocity of multiply scattered waves in strongly scattering media
}

Benoit Tallon, Thomas Brunet, Jacques Leng, John H Page

\section{To cite this version:}

Benoit Tallon, Thomas Brunet, Jacques Leng, John H Page. Energy velocity of multiply scattered waves in strongly scattering media. Physical Review B, 2020, 101 (5), 10.1103/physrevb.101.054202 . hal-03052370

\section{HAL Id: hal-03052370 \\ https://hal.science/hal-03052370}

Submitted on 10 Dec 2020

HAL is a multi-disciplinary open access archive for the deposit and dissemination of scientific research documents, whether they are published or not. The documents may come from teaching and research institutions in France or abroad, or from public or private research centers.
L'archive ouverte pluridisciplinaire HAL, est destinée au dépôt et à la diffusion de documents scientifiques de niveau recherche, publiés ou non, émanant des établissements d'enseignement et de recherche français ou étrangers, des laboratoires publics ou privés. 


\title{
Energy velocity of multiply scattered waves in strongly scattering media
}

\author{
Benoit Tallon, ${ }^{1,2, *}$ Thomas Brunet, ${ }^{1, \dagger}$ Jacques Leng $\odot,{ }^{3}$ and John H. Page $\odot^{2, \sharp}$ \\ ${ }^{1} I 2 M$, Université de Bordeaux-CNRS-Bordeaux INP, Talence F-33405, France \\ ${ }^{2}$ Department of Physics and Astronomy, University of Manitoba, Winnipeg, Manitoba, Canada R3T 2N2 \\ ${ }^{3}$ Solvay, LOF, Université de Bordeaux-CNRS, Pessac F-33608, France
}

(Received 6 November 2019; revised manuscript received 23 January 2020; accepted 24 January 2020; published 10 February 2020)

\begin{abstract}
The important influence of the relative refractive index of scattering inclusions on energy transport of classical waves through disordered media is clearly demonstrated through ultrasonic experiments on monodisperse emulsions. Our ultrasonic techniques measure both the transmitted average wave field and the multiply scattered diffusive intensity, enabling a full characterization of wave transport through the media and the measurement of both the group and energy velocities over a wide range of frequencies. The emulsions were fabricated using microfluidic techniques that permit accurate control of droplet size and concentration, for droplet inclusions with very different acoustic properties relative to the yield stress fluids in which the droplets were immersed. Thus we have been able to investigate emulsions containing either "slow" fluorinated oil droplets (sound speed $v_{1}$ less than $v_{0}$ of the surrounding fluid) or "fast" liquid metallic droplets $\left(v_{1}>v_{0}\right)$. We find that the energy velocity that describes the transport of energy by the dominant diffusive waves is mainly governed by the sound speed within the scatterers, and can be either much slower or faster than any of the other wave velocities. The possibility that the energy velocity could be faster than any other wave velocity when $n_{\text {rel }}=v_{0} / v_{1}<1$ was not anticipated in previous work. These observations are successfully explained by theories that are valid for scalar waves in media containing a low concentration of scatterers, and are directly applicable to our dilute "all-fluid" emulsions. The role of droplet resonances on the behavior of the energy velocity is also demonstrated, and the mechanism leading to the large differences in the energy velocity in the two emulsion systems is elucidated through calculations of the energy density inside the droplets relative to the incident energy density.
\end{abstract}

DOI: 10.1103/PhysRevB.101.054202

\section{INTRODUCTION}

A propagating wave in a strongly scattering medium can be decomposed into two contributions, the average wave (which, as a result of configurational averaging, includes contributions from only the forward scattered wave and the incident wave) and the multiply scattered diffusive waves (which follow a multitude of apparently random paths through the medium) [1]. Because the average wave travels coherently straight through the medium in the forward direction, it will be referred to here as the ballistic wave (in common with usage that is quite often encountered in the literature, since it shares this characteristic of ballistic ("line-of-sight") transport with a wave that experiences no scattering in a uniform medium $[2,3]$ ). When the propagation distance is small (comparable with the scattering mean free path $\ell_{\mathrm{s}}$, the average distance between scattering events), this ballistic contribution is significant and can reveal important information about the scattering medium, including the phase velocity $v_{\mathrm{ph}}$ and scattering mean free path, as well as, in the case of pulsed transport, the group velocity, $v_{\mathrm{gr}}$. Hence the propagation of the average

\footnotetext{
*Present address: ISTerre, CNRS UMR 5275, Université Grenoble Alpes, 1381 rue de la Piscine, 38610 Gieres, France.

${ }^{\dagger}$ thomas.brunet@u-bordeaux.fr

¥john.page@umanitoba.ca
}

wave field can be described by an effective wave number $k$. For longer propagation distances, the ballistic wave becomes exponentially small, and the wave field is dominated by waves that have undergone many scattering events, with local directions of propagation that have become randomized. After a linear distance corresponding to several transport mean free paths $\ell^{*}$, the transport of the waves' energy density can be described by a diffusive process with characteristic diffusivity $D$ [4-8]. The wave diffusion coefficient, $D=v_{\mathrm{e}} \ell^{*} / 3$, depends not only on the transport mean free path (the average distance over which the direction of propagation is randomized) but also on the energy velocity $v_{\mathrm{e}}$, which corresponds to the local velocity of energy transport by the diffusive waves.

While there have been a number of key experimental and theoretical investigations of both the ballistic and diffusive contributions to wave transport over the past two or more decades, interesting questions remain about the extent to which the transport velocities are influenced by the phase velocity (or refractive index) of the scattering inclusions relative to the embedding medium. Experimental investigations of the group velocity in strongly scattering media have been mostly performed with acoustic (ultrasonic) waves, since the use of phase sensitive detectors in acoustics allows the average wave field to be measured accurately [2,6,9-14]. For low scatterer concentrations, theories based on the independent scattering approximation $[15,16]$ described the experimental results very well $[10,11,13,14]$, while at high concentrations, 
an effective medium theory [1,17] gave remarkably good agreement with the data [2,9]. These experiments and theories showed the significant frequency-dependent dispersion that can result from scattering by both hard and soft scatterers over a wide range of concentrations, with the dispersion being most clearly shown through the frequency dependence of the group velocity $[2,9,14]$.

Since the theoretical work of van Albada et al. [18,19], several studies of classical wave diffusion have focused on the behavior of the energy velocity in both optics [20-22] and acoustics $[6,7,14]$, paying particular attention to the resonant scattering regime. While all of these experiments, and the original theoretical predictions, showed significant decreases in the energy velocity due to scattering resonances, differences were found in the link between the group velocity and the energy velocity. In typical acoustic systems, the scattering particles are hard, with a small relative refractive index, $n_{\text {rel }}<$ 1 [23], and the group and energy velocities were found to be quite similar in magnitude [2]. However, in typical optical systems, the refractive index of the particles is high $(n>1)$, and very different behavior was predicted for these two velocities. Such large differences between $v_{\mathrm{e}}$ and $v_{\mathrm{gr}}$ were confirmed by ultrasonic experiments on so-called "resonant emulsions" for which $n_{\text {rel }}$ is also larger than 1 [14]. Thus it seems that the refractive index of the scattering particles plays an important role in the behavior of the energy velocity. However, a detailed comparison between all of these experiments is hindered by significant differences in the nature of the scattering (e.g., acoustic versus optical scattering, solid scatterers versus fluid scatterers in acoustics), motivating a fresh examination of the influence of refractive index on the behavior of the energy velocity. The ideal comparison would involve experiments on samples in which only the relative refractive index of the scatterers is varied.

In this paper, we follow this "ideal" path by examining the role of the scatterers' sound speed on the energy velocity by performing ultrasonic experiments on two carefully chosen model systems. Our model systems are emulsions in which the droplet materials have very different acoustic velocities. The all-fluid structure of our samples means that only scalar acoustic waves propagate, enabling reliable comparisons with theories for the energy velocity which have been developed for scalar waves $[18,19]$. Furthermore, we study samples with low concentrations of scatterers for which theoretical models based on the independent scattering approximation are expected to be accurate [16]. Our emulsion samples are made either from fluorinated FC40 oil droplets $\left(n_{\text {rel }}=2.3\right)$ suspended in a water-based yield-stress fluid (diluted hair gel) or from GaIn droplets ( $n_{\text {rel }}=0.56$ ) suspended in another water-based yield-stress fluid (diluted solution of xanthan gum in water). The intrinsic losses of these materials are very low in the $\mathrm{MHz}$ range so that the effects of multiple scattering on wave transport are not masked by absorption. Through choice of droplet size and ultrasonic frequency, we are able to study wave transport in the intermediate frequency regime $\left(k_{0} a>1\right.$, with $k_{0}$ the wave number in the pure gel and $a$ the radius of the droplets), where strong scattering occurs and may be further enhanced by "shape" resonances of the droplets (analogous to Mie resonances in optics but for scalar waves in our acoustic systems). In this regard, a first look

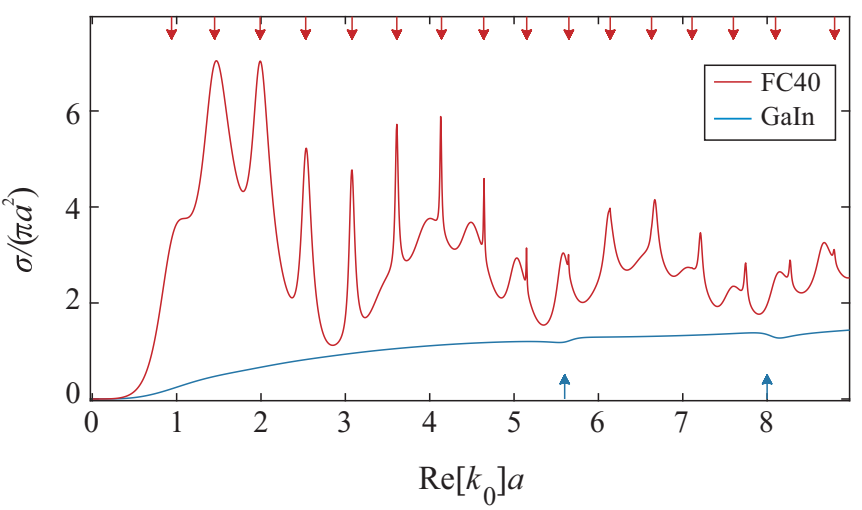

FIG. 1. Scattering cross section of a single droplet of FC40 oil (red solid line) and GaIn alloy (blue solid line) both immersed in water. Red and blue arrows indicate resonant frequencies for FC40 and GaIn droplets, respectively.

at the scattering properties of our droplets can be gleaned by comparing calculations of their scattering cross sections, which are shown in Fig. 1. For these systems, the scattering cross section for a single droplet is either comparable to or much greater than the geometric cross section $\left(\pi a^{2}\right)$ in the intermediate frequency regime, confirming that the scattering is indeed strong. Furthermore, this figure shows striking differences in the impact of resonances on the scattering for the two types of droplets. For the FC40 oil droplet, its low sound speed results in strong, low frequency resonances whereas, for the GaIn droplets, the resonances occur at higher frequencies and are weak-barely visible in the figure. This basic comparison of the scattering properties of the droplets already suggests that one might expect significant differences in the energy velocity for wave transport through suspensions of these droplets.

The outline of the paper is as follows. The fabrication method for producing the samples and ultrasonic measurement procedures are described in Sec. II. Two ultrasonic setups are used in order to measure the average wave and the diffuse parts of the transmitted wave field. In both cases, pulsed experiments are used. Thus we are able to directly compare the energy velocity of the multiply scattered waves with the group velocity that characterizes the transmission of the average wave field in pulsed experiments. Section III presents the experimental results for scalar wave propagation in our dilute samples and the comparison of experiment with theory. For the coherent ballistic waves, the experimental results are compared to calculations performed using the independent scattering approximation (ISA), which we show gives accurate predictions for the scattering properties of the samples. In particular, we contrast the behavior of the group velocity for the two samples, confirming that it remains well defined even when the dispersion due to scattering is strong. For the diffuse waves, the transport of energy through the samples is well described by the diffusion approximation, enabling the diffusion coefficient to be measured and compared with theory. We show how these measurements can be used to determine the energy velocity. The rest of this section is dedicated to discussions and theoretical descriptions of the energy velocity, including the scattering delay induced 

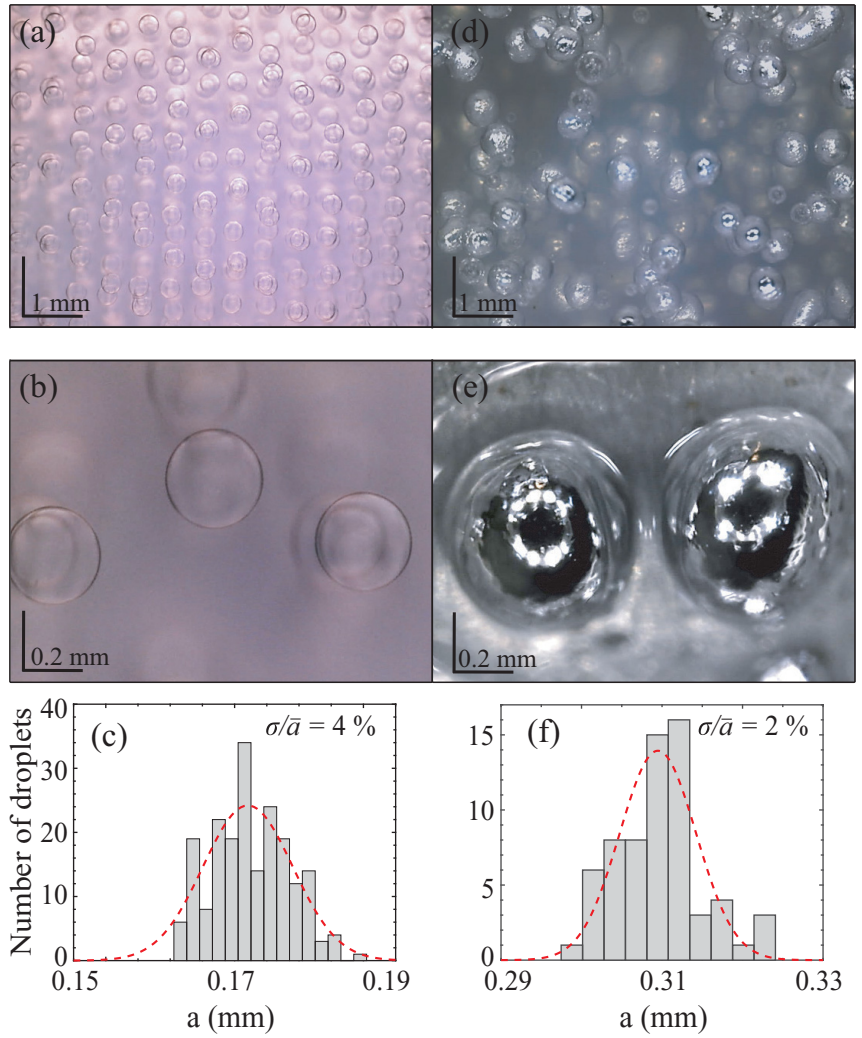

FIG. 2. Fluorinated oil droplets (a) and (b) as well as GaIn droplets (d) and (e) suspended in the yield-stress fluids. Panel (a) shows the droplets immediately after injection; they are well mixed during transfer to the ultrasonic measurement cell to ensure that their positions are disordered. The lens magnification was $\times 50$ for images (a) and (d) and $\times 250$ for (b) and (e). The size distributions obtained by optical methods are plotted in (c) for the FC40 droplets and (f) for the GaIn droplets (the red dashed lines represent the Gaussian fits used to assess the droplet polydispersity).

by energy trapped in the particles in the resonant regime. The comparison of the results for the two samples shows very convincingly that the phase velocity inside the scatterers not only affects the magnitude of the scattering resonances but also influences the energy velocity significantly, even at frequencies for which the scattering is not affected by resonances.

\section{EXPERIMENTS}

\section{A. Sample fabrication}

Our "resonant emulsions" [Figs. 2(a) and 2(b)] are made from fluorinated oil (FC40) droplets (with sound speed $v_{1}=$ $0.64 \mathrm{~mm} / \mu \mathrm{s}$, density $\rho_{1}=1.85 \mathrm{~g} / \mathrm{cm}^{3}$, and amplitude attenuation coefficient due to absorption $\alpha_{1}=10^{-3} f^{2} \mathrm{~mm}^{-1}$ at frequency $f$ in $\mathrm{MHz}$ ) randomly dispersed in a yield-stress fluid consisting of diluted hair gel $\left(v_{0}=1.48 \mathrm{~mm} / \mu \mathrm{s}, \rho_{0}=\right.$ $1 \mathrm{~g} / \mathrm{cm}^{3}$, and $\alpha_{0}=5 \times 10^{-5} f^{2} \mathrm{~mm}^{-1}$ ). This yield-stress fluid has a small nonzero shear modulus that is sufficient to prevent the droplets from sedimenting [24], but frequency-dependent rheological behavior that makes its acoustic properties similar to those of water at the megahertz frequencies used in our ultrasonic experiments [11]. For convenience, the main
TABLE I. Properties of the FC40 and GaIn emulsion samples. The sample thickness and slab diameter are for the diffusion coefficient measurements only; thinner samples were used for the average wave measurements so that the ballistic pulses could be clearly resolved.

\begin{tabular}{lcc}
\hline \hline & FC40 emulsion & GaIn emulsion \\
\hline Droplet $v_{1}(\mathrm{~mm} / \mu \mathrm{s})$ & 0.64 & 2.74 \\
Droplet $\rho_{1}\left(\mathrm{~g} / \mathrm{cm}^{3}\right)$ & 1.85 & 6.28 \\
Host matrix $v_{0}(\mathrm{~mm} / \mu \mathrm{s})$ & 1.48 & 1.52 \\
Host matrix $\rho_{0}\left(\mathrm{~g} / \mathrm{cm}^{3}\right)$ & 1.00 & 1.00 \\
Droplet radius $\bar{a}(\mathrm{~mm})$ & 0.17 & 0.31 \\
Volume fraction $\phi(\%)$ & 5 & 16 \\
Polydispersity $P(\%)$ & 4 & 2 \\
Sample thickness $L(\mathrm{~mm})$ & 26 & 12 \\
Slab diameter $D(\mathrm{~mm})$ & 94 & 50 \\
\hline \hline
\end{tabular}

properties of both constituents are summarized in Table I. Microfluidic techniques [11,13] are employed in order to fabricate an almost monodisperse population of droplets: a moving syringe (at constant velocity $V=35 \mathrm{~mm} / \mathrm{s}$ ) continuously injects the oil (at constant flow rate $Q=35 \mu \mathrm{L} / \mathrm{min}$ ) within the water-based gel matrix via a thin needle with an inner radius that is approximately equal to the droplets' mean radius $\bar{a}=0.17 \mathrm{~mm}$. The size of the droplets can easily be chosen by setting the needle velocity $V$ and the flow rate $Q$ of the injection. Displacements and flow rates are controlled by robotics, which ensures the very low standard deviation $\sigma$ of the droplets' size distribution. Indeed, with this method, the polydispersity $P=\sigma / \bar{a}$ is about $4 \%$ [Fig. 2(c)] in all experiments, which ensures that sharp resonances can be detected [25], since most of the droplets have the same size and thus the same resonant frequency.

In comparison with the relatively well studied FC40 emulsions $[13,14,25]$, the fabrication of GaIn droplet suspensions [Figs. 2(d) and 2(e)] is more challenging. Gallium-indium eutectic alloy $\left(v_{1}=2.74 \mathrm{~mm} / \mu \mathrm{s}, \rho_{1}=6.28 \mathrm{~g} / \mathrm{cm}^{3}\right.$, and low absorption [26]) was purchased from the Indium Corporation and droplets of this alloy were prepared using a microfluidic chip made of glass capillaries [27]. This eutectic alloy remains liquid at room temperature and is thus suitable for fabrication of emulsions. In this chip, the coflowing geometry permits us to generate droplets of GaIn in a carrier fluid made of a mixture of water and polyvinylalcohol (PVA, 1\% in mass, with a molar mass of $70000 \mathrm{~g} / \mathrm{mol}$ and $98 \%$ hydrolysis). The final capillary, with an inner diameter of $\sim 600 \mu \mathrm{m}$, fixes the diameter of the GaIn droplets and is surface-treated with a hydrophilic polymer coating (Mirapol Surf S 500 from Solvay) in order to prevent surface fouling. Large flow rates of about $1 \mathrm{~mL} / \mathrm{min}$ for each fluid yield a large number of droplets in a matter of minutes. The droplets are characterized using optical microscopy; they have a radius of $\sim 0.31 \mathrm{~mm}$ with a polydispersity $P \sim 2 \%$ [Fig. 2(f)]. Eventually, these droplets are collected "on-the-fly" in a large $(\sim 200 \mathrm{~mL})$, slowly stirred, maturing bath that contains water and PVA and where we measured the dissolved dioxygen content $(\sim 10$ to $20 \mathrm{ppm}$ ). This agent is of prime importance to oxidize the surface of the droplets [28] and make the surface rigid enough to enable the droplets to be redispersed in a yield- 


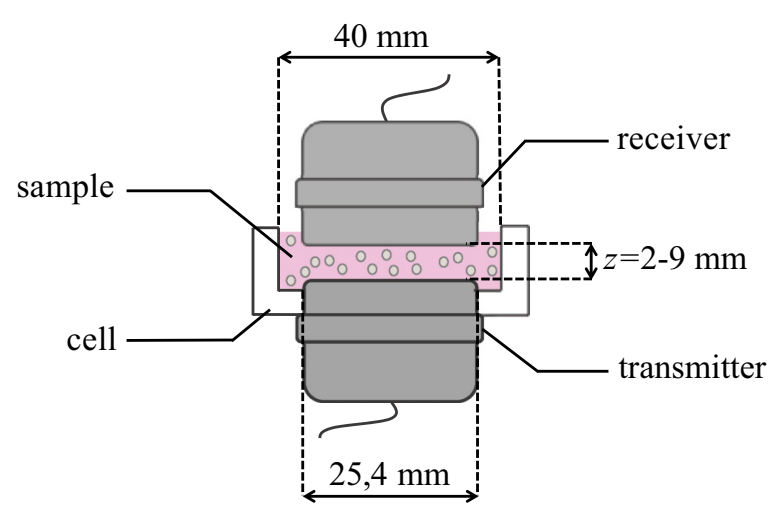

FIG. 3. Experimental setup for measuring the average wave field transmitted through the emulsion samples.

stress fluid with negligible coalescence or deformation. For the GaIn droplet emulsions, a dilute solution of xanthan gum in water was used as the matrix, since the acidity of hair gel makes it unsuitable, as it attacks the thin nanometric oxide layer on the GaIn surface (thickness $\sim$ few nanometers [29]). Consequently, the sound speed in the matrix for the GaIn emulsions is slightly higher than for the FC40 emulsions (Table I), but the absorption in this matrix is still expected to be very small and similar to that of the FC40 emulsions.

\section{B. Average transmitted wave field}

In order to measure the average wave field that has traveled through our samples, two broadband ultrasonic transducers, with a central frequency of $5 \mathrm{MHz}$, were placed on opposite sides of, and in direct contact with, the emulsion (Fig. 3). In this configuration, near field diffraction effects due to the finite size of the transducers may lead to an overestimation of the dispersion of the sample. In our case these effects were estimated by following the method described in Ref. [30] and were found to be negligible (the relative error induced by near field effects on the attenuation and phase velocity measurements is about 1\%). With this setup, the average part of the transmitted wave was measured by taking advantage of the spatial averaging of the acoustic field across the large surface area of the receiver (1-in. diameter, corresponding to a spatial average over more than 1500 speckles at $2.5 \mathrm{MHz}$ ). Furthermore, the propagation distance $(z=2 \mathrm{~mm}$ for the FC40 emulsion and $z=9 \mathrm{~mm}$ for the GaIn emulsion) is small enough to ensure that the average wave field was not too attenuated by scattering effects. The wave number $k$ of the ballistic pulse was obtained from a differential measurement between two acquisitions: a reference signal, measured through pure gel matrix, and the average transmitted signal through the sample (Fig. 4).

For both emulsions, dispersion and/or frequencydependent attenuation caused the shape of the transmitted pulses to differ from the reference pulses. For example, in Fig. 4(a), the tail on the transmitted pulse, which shows up as a small secondary pulse occurring around $3 \mu \mathrm{s}$, is a signature of the strong dispersion in the FC40 emulsion caused by droplet resonances, and is not due to an echo between the transducers. Scattering effects induced by these resonances
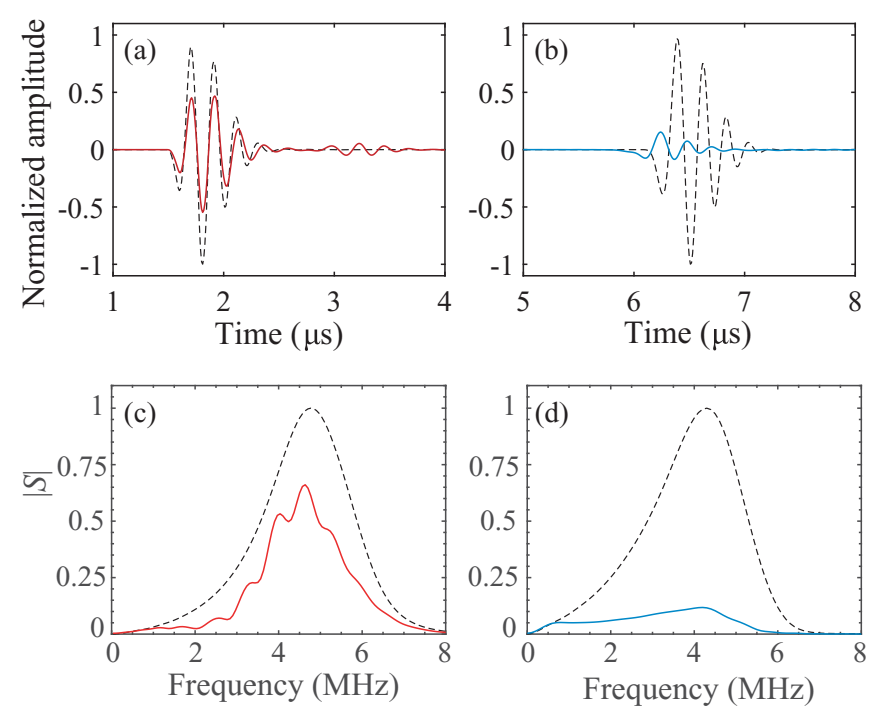

FIG. 4. Transmitted signals through $2 \mathrm{~mm}$ of FC40 emulsion (a) and $9 \mathrm{~mm}$ of GaIn suspension (b). The black dashed lines represent the reference signals in the yield-stress fluid host matrices for these two cases. Plots (c) and (d) represent the magnitude of the corresponding Fourier transforms for FC40 and GaIn samples, respectively (where the black dashed lines are Fourier transforms of the reference signals).

are also responsible for attenuation peaks, which can be identified by dips in the average signal spectrum [Fig. 4(c)]. Neither of these resonance effects is visible in Figs. 4(b) and 4(d), indicating how very weak the GaIn droplet resonances are compared with those of the FC40 droplets. Note that the difference in the overall magnitude of the pulses (and their Fourier transforms) for the two emulsions is also significantly influenced by the difference in propagation distances for these measurements.

From these measurements of the average wave pulses, we extract the phase velocity $v_{\mathrm{ph}}=\omega / \operatorname{Re}[k]$ and extinction length $\ell_{\text {ext }}=1 /(2 \operatorname{Im}[k])$ using a "spectral division" method:

$$
\begin{aligned}
& \ell_{\mathrm{ext}}=\left[2 \ln \left(\frac{\left|S_{\mathrm{ref}}\right|}{|S|}\right) / z\right]^{-1}, \\
& v_{\mathrm{ph}}=\left(\frac{\varphi-\varphi_{\mathrm{ref}}}{\omega z}+\frac{1}{v_{0}}\right)^{-1},
\end{aligned}
$$

where $S_{\text {ref }}=\left|S_{\text {ref }}\right| e^{i \varphi_{\text {ref }}}$ and $S=|S| e^{i \varphi}$ represent the Fourier transforms of the reference and average transmitted pulses, respectively (Fig. 4). (Note that the phase of the ultrasonic signals is represented by the symbol $\varphi$, which should be distinguished from $\phi$ that denotes volume fraction.) This method allows direct measurement of the extinction length and phase velocity over a wide frequency range (from $f=1 \mathrm{MHz}$ to $f=8 \mathrm{MHz}$ ), so that a large set of droplet resonances can be observed for the FC40 emulsions. The extinction of the ballistic pulse during its propagation has two origins: the attenuation by scattering and the absorption due to the intrinsic viscosity of the fluids. The expression for the extinction length is thus

$$
\frac{1}{\ell_{\mathrm{ext}}}=\frac{1}{\ell_{\mathrm{s}}}+\frac{1}{\ell_{\mathrm{a}}},
$$




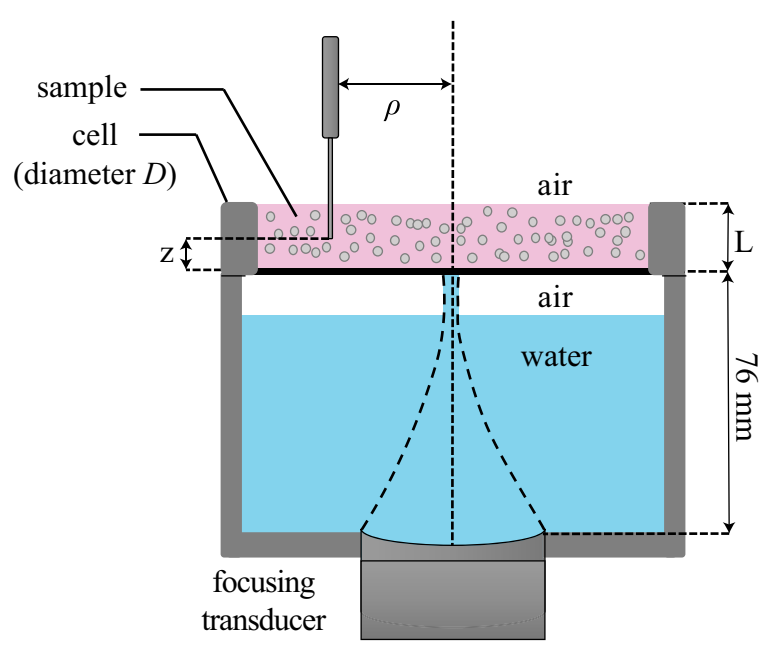

FIG. 5. Representation of the experimental setup used to measure the time- and position-resolved transport of multiply scattered acoustic waves inside the samples.

where $\ell_{\mathrm{s}}$ is the scattering mean free path (the characteristic attenuation distance of the ballistic intensity due to scattering) and $\ell_{\mathrm{a}}$ is the absorption length which represents the losses. For the fluids considered here, we can be confident that $\ell_{\text {ext }} \sim$ $\ell_{\mathrm{s}}$, since the absorption length $\ell_{\mathrm{a}}$ is estimated to be typically about 100 times larger than $\ell_{\mathrm{s}}$ over the frequency range of the measurements [14]. The extinction of the average wave during its propagation is thus almost entirely due to scattering rather than viscous dissipation in the fluids.

With this ballistic pulse measurement, we can also measure the group velocity. The transmitted signals through the emulsions and through the pure matrix reference medium were narrow-band filtered (50 kHz FWHM bandwidth) around each frequency of interest in order to obtain Gaussian pulses [2,9]. The time of flight of the peaks of the pulse envelopes, $t_{\mathrm{pk}}$ and $t_{\mathrm{pk}, \text { ref }}$, then gives the group time of emulsion and reference signals, apart from a possible shift due to a likely difference between the trigger and the initial time at which the pulses entered each medium. This shift was determined from the difference between the peak arrival time of the reference pulse through the pure matrix and its known group time, which is given by $z / v_{0}$, as the matrix is nondispersive. The group velocity $v_{\mathrm{gr}}$ of the emulsions was then determined from

$$
v_{\mathrm{gr}}=\left(\frac{t_{\mathrm{pk}}-t_{\mathrm{pk}, \mathrm{ref}}}{z}+\frac{1}{v_{0}}\right)^{-1} .
$$

Repeating the process for many filtering frequencies within the bandwidth of the unfiltered pulse yields frequencyresolved measurements of the group velocity.

\section{Multiply scattered waves}

The experimental setup used to probe the propagation of the multiply scattered waves is illustrated in Fig. 5. In order to measure the total time-dependent transmitted field in the regime where multiple scattering dominates, the propagation distance was increased $(z=20 \mathrm{~mm}$ for the FC40 emulsion and $z=10 \mathrm{~mm}$ for the GaIn suspension), and the large receiver was replaced by a small needle hydrophone (with

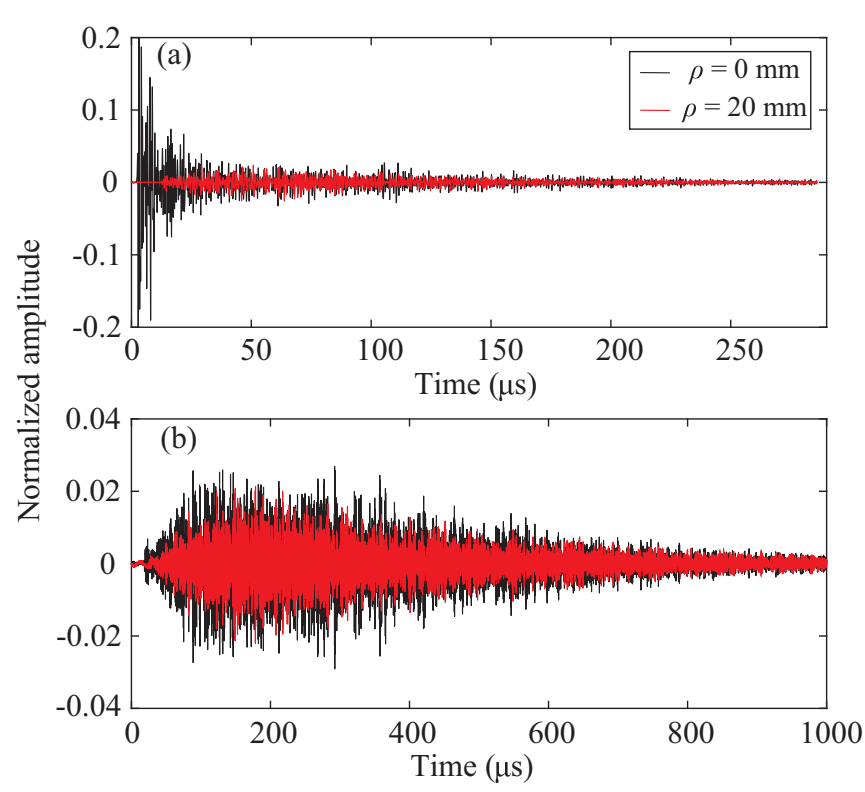

FIG. 6. Transmitted unfiltered coda (for two transverse distances $\rho$ ) at a depth $z=10 \mathrm{~mm}$ within the GaIn suspension (a) and $z=$ $20 \mathrm{~mm}$ within the FC40 emulsion (b).

a diameter of $500 \mu \mathrm{m}$, which is approximately equal to the acoustic wavelength). The hydrophone was immersed in the emulsion and, because of its small size, was capable of resolving the spatial variation of the acoustic wave field inside each sample. In these experiments, the incoming wave was generated by a focusing transducer and was further confined to a well localized pointlike source by an acoustic diaphragm, which was placed on one side of a circular slab containing the sample. This acoustic diaphragm consisted of an air slab, which acts as an opaque barrier for $\mathrm{MHz}$ ultrasound, with a soft-solid cylinder in the center, which acts as a transparent aperture. The cylindrical aperture had a diameter of about $5 \lambda_{0}$ (the wavelength in the pure matrix at $f=2.5 \mathrm{MHz}$ ), and was made from PDMS (polydimethylsiloxane). The input signal was a short Gaussian pulse (centered around $2.5 \mathrm{MHz}$ ). The hydrophone detector was attached to motorized stages, allowing the diffuse field to be probed at any point $(\rho, \theta, z)$ within the circular slab (where $\rho=z=0 \mathrm{~mm}$ denotes the midpoint of the source at the input face of the emulsion).

Figure 6 shows the time dependence of the transmitted fields for the two samples at different transverse positions $\rho$ within the slab. These long coda signals, which arrive after the (much smaller) ballistic pulse, are characteristic of multiple scattering contributions, since they extend over a range of times that greatly exceeds the width of the input pulse (200 to 1000 times longer than the input pulse width for the two emulsion samples investigated). Furthermore, the slow decrease in the amplitude of these signals with time is a signature of low absorption, due to very low intrinsic acoustic losses in the fluids employed to fabricate the samples.

To characterize the diffusive transport of energy by the multiply scattered waves, the average field (averaged over all transverse positions for a given $z$ ) was first subtracted from the total transmitted field in order to suppress any residual ballistic component and obtain only the multiply scattered 



FIG. 7. On the top: unfiltered transmitted speckle patterns (spatial variation of the energy density for a value of $z$ just inside the output surface of the sample, normalized by the energy density at the center of the pattern) measured at three different times in a FC40 emulsion. On the bottom: angular-averaged spatial profiles of the energy density at the same value of $z$ (also normalized by the energy density at $\rho=0 \mathrm{~mm}$ ). Red curves are fits of the Gaussian expression $\exp \left[-\rho^{2} /(4 D t)\right]$ to the data and the arrows indicate the half width at half maximum $[=w(t) / \sqrt{\ln 2}]$ of the diffuse halo.

field (the $\operatorname{cod} a$ ). The envelopes of the time-dependent transmitted fields were then calculated and squared to determine a quantity proportional to the energy density at the position of the hydrophone $z$ inside the sample. If the propagation distance is large enough $\left(\gg \ell^{*}\right)$ [4], the ensemble-averaged energy density obeys the diffusion equation with characteristic diffusion coefficient $D$. From the solution of the diffusion equation for a point source incident on an infinite slab of thickness $L$, the evolution of the energy density as a function of time and position is known to have the form $U(z, \rho, t)=$ $U(z, 0, t) \exp \left[-\rho^{2} /(4 D t)\right]$, where $U(z, 0, t)$ is a relatively complicated function that includes the effects of boundary conditions and absorption [5,31]. For a given value of $z$, the ratio $R(\rho, t)=U(\rho, t) / U(0, t)$ is a Gaussian that describes the transverse growth of the diffusive halo. To illustrate experimentally the behavior of the halo for one of our emulsions, we show in Fig. 7 (top panels) the energy density $U(\rho)$ of the transmitted speckle pattern at three different times $t$, normalized by its values for the on-axis $(\rho=0 \mathrm{~mm})$ position. While the top panel of Fig. 7 reveals the characteristic spatial fluctuations of speckle patterns, the angular averaged energy density profiles shown in the lower panels clearly show the smooth Gaussian shape of the ensemble averaged diffuse halo. Note that good ensemble averaging was achieved not only by angular averaging over many different positions of the hydrophone (thereby probing different regions of the sample) but also by stirring the emulsion via the movement of the hydrophone from one position to another inside the sample. The fits of Gaussians to these averaged spatial profiles (red solid curves) then enable the diffusivity $D$ to be measured, and give consistent results for the three times at which the profiles are shown. Thus the measurement of the dynamic transverse spatial profile $R(\rho, t)$ enables $D$ to be measured, and this approach has the advantage of avoiding the complications of boundary conditions and absorption that influence the full profile $U(z, \rho, t)[5]$.

To measure the diffusion coefficient as a function of frequency, the transmitted time-dependent field $(\operatorname{cod} a)$ at each position of the hydrophone was filtered numerically with a narrow-band Gaussian filter $(\Delta f=20 \mathrm{kHz})$ about several central frequencies before determining the normalized average energy density ratio $R(\rho, t)$. The procedure for accurately measuring $D$ was to capitalize on the linear dependence of the width squared of the diffuse halo on time, $w^{2}(t)=$ $-\rho^{2} / \ln [R(\rho, t)]=4 D t$, and to perform a linear least squares fit of $w^{2}(t)$ versus $t$ so as to determine $D$ from the slope. The procedure was repeated for several different $\rho$ values to reduce the measurement uncertainty. This fit was performed for the range of times $\Delta t$ over which numerical simulations of diffusion in a slab show that lateral walls of the cell have no effect on the evolution of the halo.

\section{RESULTS AND DISCUSSION}

\section{A. Effective wave number}

The propagation of the average field is determined by the effective wave number $k$, which is directly related to the phase velocity and extinction length introduced in Sec. II B:

$$
k=\frac{\omega}{v_{\mathrm{ph}}}+i \frac{1}{2 \ell_{\mathrm{ext}}} .
$$

The frequency dependence of the effective wave number may be readily calculated theoretically using the ISA, thereby enabling our experimental data to be compared with theory. The theoretical expression for the wave number, taking into account the size distribution of the droplets, is [25]

$$
k^{2}=k_{0}^{2}+\int_{a} 4 \pi \eta_{a} f(0) d a,
$$

where $\eta_{a}$ is the Gaussian size distribution of the droplets: $\eta_{a}=\eta /(\sqrt{2 \pi} \sigma) e^{-(a-\bar{a})^{2} / 2 \sigma^{2}}$, with $\eta$ being the average droplet concentration. In the far field approximation, the scattering function $f(\theta)=|f(\theta)| \exp \left[i \varphi_{s}(\theta)\right]$, with magnitude $|f(\theta)|$ and phase $\varphi_{s}(\theta)$, represents the scattering amplitude in the direction given by the angle $\theta$ with respect to the incident wave vector, and is given by the following expression:

$$
f(\theta)=\frac{1}{i k_{0}} \sum_{\mathrm{n}}(2 \mathrm{n}+1) A_{\mathrm{n}} P_{\mathrm{n}}(\cos \theta) .
$$

Here $P_{n}$ denote the Legendre polynomials and $A_{\mathrm{n}}$ are the amplitude coefficients of the scattered field, given by Eq. (A4) $[25,32]$. From Eqs. (4) and (5), we directly obtain $v_{\mathrm{ph}}$ and $\ell_{\mathrm{s}}=\ell_{\mathrm{ext}}$ (when no dissipative effects are taken into account), and calculate $v_{\text {gr }}$ from the numerical derivative of $\partial \omega / \partial k$.

For the FC40 emulsion, the strong impact of droplet resonances on the acoustic properties is clearly visible in our experimental data for the extinction length, phase and group velocities, as reported in Fig. 8. Notably, at the resonant frequencies of a single droplet, there are dips in the scattering mean free path, rapid increases in the phase velocity, and sharp peaks in the group velocity [25]. Excellent agreement is found between these measurements and theoretical predictions based on the ISA. It is worth noting that the excellent 

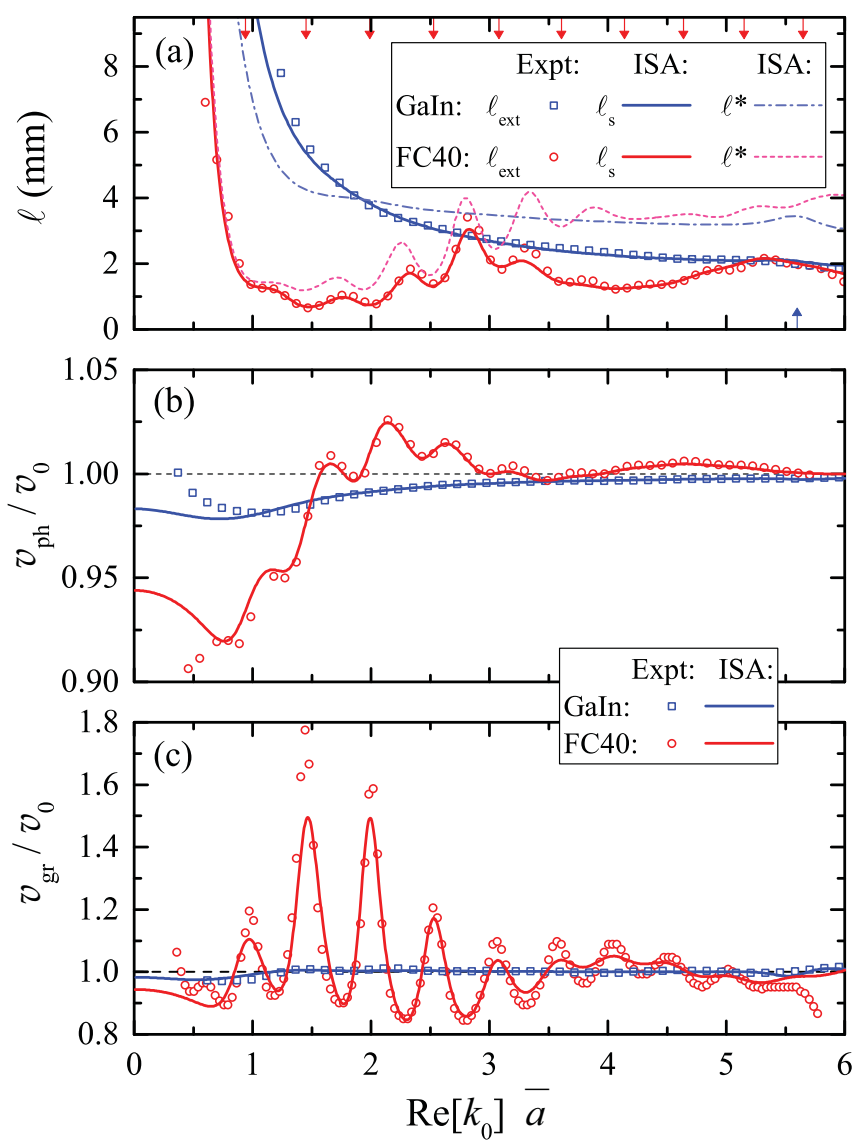

FIG. 8. Measured (a) extinction length $\ell_{\text {ext }}$ (essentially equal to the scattering mean free path), (b) phase $v_{\text {ph }}$, and (c) group velocities $v_{\mathrm{gr}}$ versus frequency for the two different samples (shown by red circles for the FC40 emulsion and blue squares for the GaIn suspension). Independent scattering approximation predictions are represented by solid lines for the volume fractions $\phi_{\mathrm{FC} 40}=5 \%$ and $\phi_{\mathrm{GaIn}}=16 \%$ and the polydispersities $P_{\mathrm{FC} 40}=4 \%$ and $P_{\mathrm{GaIn}}=2 \%$. Velocities are normalized by the sound speed in the pure matrix $v_{0}$. Resonant frequencies are identified by red arrows for a FC40 droplet with $a=0.17 \mathrm{~mm}$ and blue arrows for a GaIn droplet with $a=0.31 \mathrm{~mm}$ when both are immersed in their respective yield stress fluids. The arrows delineating the resonant frequencies correspond to frequencies for which the amplitude of the wave field is a maximum inside the droplets, as determined by the coefficients $B_{n}$ given by Eq. (A5). The dashed lines in (a) represent ISA predictions for $\ell^{*}$ as discussed in the text (Sec. III B, third paragraph).

agreement between the experimental measurements of the extinction length and the ISA predictions for the scattering mean free path further confirm that dissipation in these samples is very small (virtually negligible for coherent wave propagation straight through the samples). These theoretical predictions take into account the size distribution of the droplets, which, while quite narrow, as noted in Sec. II A, is still sufficient to blur somewhat the sharp features in these curves due to the dependence of resonant frequency on droplet radius [25]. Nonetheless, the scattering resonances of the FC40 droplets are sufficiently strong that distinct minima in $\ell_{\mathrm{s}}$ and peaks in $v_{\text {gr }}$ remain very clearly resolved. By contrast, for the suspension of GaIn droplets, the resonances are so much
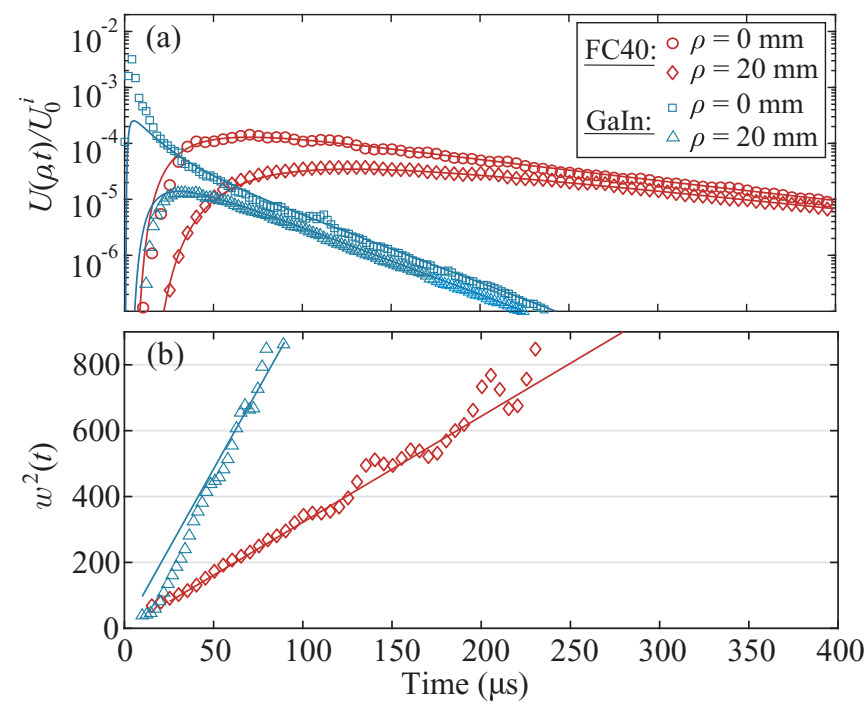

FIG. 9. (a) Time dependence of the ensemble-averaged energy density of the acoustic field at position $z$ inside the samples as a function of time (time-of-flight curves), normalized by the incident energy density $U_{0}^{i}$ (from a pointlike source in both time and space). These data are for a filtering frequency $f=3.0 \mathrm{MHz}$ and for two different transverse distances $\rho$. The measurement positions $z$ are the same as those given in the caption to Fig. 6 . The experimental data for the two samples are represented by open symbols. The solid lines represent the results of numerical simulation of diffusion for a point source in a slab with the geometry of our experimental setup (Fig. 5). Measured diffusivity $D$ and calculated transport mean free path $\ell^{*}$ are used as inputs for this simulation. (b) Time dependence of the width squared $w^{2}(t)$ of the diffusive halo deduced from the measured ratio $U(\rho, t) / U(0, t)$ of the time-of-flight curves (symbols). The solid lines represent the linear fits $w^{2}(t)=4 D t$ used to extract the diffusion coefficient $D$ from these measurements.

weaker that their effect on the frequency dependence of the acoustic velocities and mean free path is washed out by the weak polydispersity and, even close to the first resonance of the GaIn droplets at $\operatorname{Re}\left[k_{0}\right] a=5.6$, no obvious structure in the data is visible (Fig. 8).

\section{B. Diffusion coefficient and transport velocity}

The experimental results for the propagation of the average wave through the two samples (Sec. III A) demonstrate that these emulsions have large scattering strengths. In particular, in the case of the FC40 emulsion, the scattering mean free path $\ell_{\mathrm{s}}$ is approximately equal to the wavelength in the pure matrix $\lambda_{0}$. For the GaIn suspension, the mean free path is larger but remains sufficiently small $\left(\ell_{\mathrm{s}} \sim 4-5 \lambda_{0}\right)$ for multiple scattering effects to be observed. As discussed in Sec. II C, the transport of energy by the multiply scattered coda waves can be described using the diffusion approximation, which gives simple predictions for the time dependence of the transmitted average energy density and for the transverse growth of the diffuse halo. Typical results at $3.0 \mathrm{MHz}$ are shown in Fig. 9 for both samples. Figure 9(a) shows the temporal evolution of the average energy density, normalized by the incident energy density $U_{0}$, for two transverse distances $\rho=0 \mathrm{~mm}$ and $\rho=20 \mathrm{~mm}$, while Fig. 9(b) illustrates the increase in 


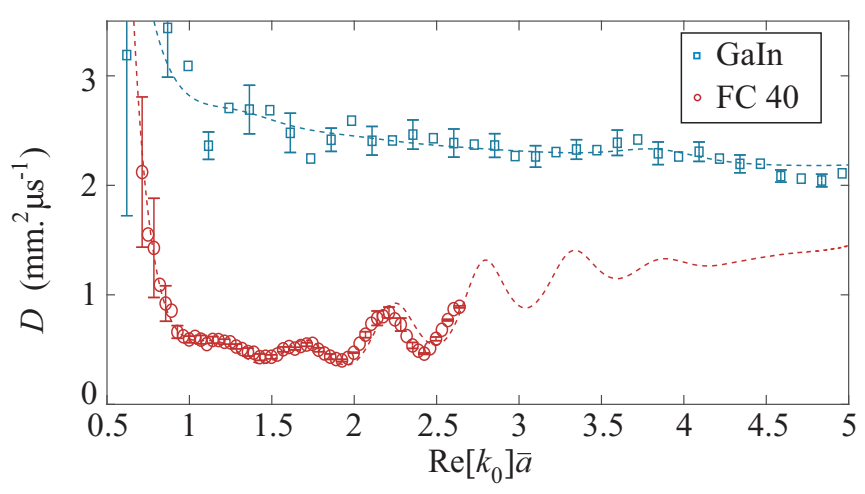

FIG. 10. Measured diffusion coefficients versus frequency for the GaIn and FC40 emulsions (open blue squares and red circles, respectively). The dashed lines represent theoretical calculations for $D=v_{\mathrm{e}} \ell^{*} / 3$, using the predictions for $\ell^{*}$ (Fig. 8) and $v_{\mathrm{e}}$ [Eqs. (7) and (8)] discussed in the text.

the transverse width squared $w^{2}(t)$ with time. Both graphs demonstrate that diffusion in the GaIn sample happens much more rapidly. This difference is quantified by the slopes of the linear least squares fits of $w^{2}(t)$ versus time [solid lines in Fig. 9(b)], from which we determine that the diffusion coefficients are $D_{\mathrm{FC} 40}=0.81 \pm 0.01 \mathrm{~mm}^{2} \mu \mathrm{s}^{-1}$ for the FC40 emulsion and $D_{\mathrm{GaIn}}=2.42 \pm 0.09 \mathrm{~mm}^{2} \mu \mathrm{s}^{-1}$ for the GaIn suspension at this frequency. Because of the finite transverse width of our slab-shaped samples, a numerical solution of the diffusion equation, taking into account this difference in sample shape from that of an infinite slab, was used to generate the theoretical curves for $U(\rho, t) / U_{0}$ shown in Fig. 9(a). In these calculations, the diffusive source position was $z^{\prime}=\ell^{*}$, the internal reflection coefficient at the interface between the emulsions and air was calculated to be 0.999 , and the absorption time $\tau_{a}=65 \mu \mathrm{s}$ for GaIn and $\tau_{a}=180 \mu \mathrm{s}$ for FC40.

By repeating this procedure for measuring $D$ from $w^{2}(t)$ at different filtering frequencies (Sec. IIC), we obtain the experimental values of the diffusion coefficient shown as a function of frequency in Fig. 10. As might be anticipated from the typical results in Fig. 9 at $3 \mathrm{MHz}$, Fig. 10 shows that $D$ for the GaIn suspension is much larger than for the FC40 emulsion throughout the entire frequency range of the measurements. Also, the variation of $D$ with frequency for the GaIn sample is quite smooth, whereas the influence of the sharp resonances for FC40 droplets is reflected in the much greater variation with frequency of $D$ for the FC40 emulsion. The natural question to ask is whether these differences in $D=v_{\mathrm{e}} \ell^{*} / 3$ are mainly due to differences in the mean free paths or whether these differences also indicate significant differences in the behavior of the energy velocity for these two systems.

To answer this question, we first calculate the frequency dependence of $\ell^{*}$ within the ISA using the well-established expression $\quad \ell^{*}=\ell_{\mathrm{S}} /(1-\langle\cos \theta\rangle)$, where $\langle\cos \theta\rangle=$ $\int_{0}^{\pi} \cos \theta|f(\theta)|^{2} \sin \theta d \theta / \int_{0}^{\pi}|f(\theta)|^{2} \sin \theta d \theta$ is the weighted average of $\cos \theta$ over scattering angles $\theta[1,33]$. The reliability of this $\ell^{*}$ estimation is expected to be very good and is supported by the excellent agreement between the ISA model

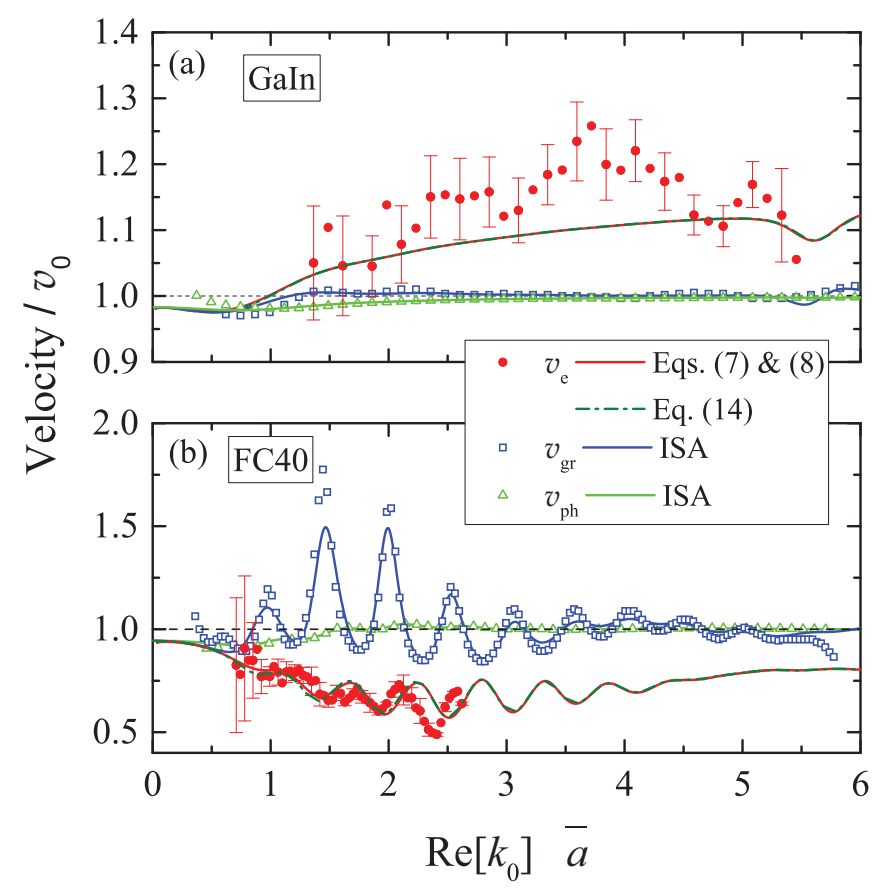

FIG. 11. Frequency dependence of the measured energy velocity in (a) GaIn and (b) FC40 (solid red circles). To highlight the important differences in the energy velocities in these samples, the energy velocities are compared with our data for the phase and group velocities from Figs. 8(b) and 8(c). Theoretical predictions are shown by the solid and dashed lines, as indicated in the legend. Results are normalized by the sound speed in the pure matrix $v_{0}$.

and our experimental data for the scattering mean free path [Fig. 8(a)]. These results for $\ell^{*}$ are plotted along with $\ell_{\mathrm{s}}$ in Fig. 8(a), and show substantial differences in $\ell^{*}$ between the two systems at low frequencies but much smaller differences at higher frequencies.

From these results for $\ell^{*}$, the measurements of $D$, and the relation $v_{\mathrm{e}}=3 D / \ell^{*}$, we determine the energy velocity $v_{\mathrm{e}}$ as a function of frequency for the two samples, and compare their values in Fig. 11 with the phase and group velocity measurements. Strikingly, this figure shows that the energy velocity for the GaIn emulsion, for which the acoustic phase velocity of the droplets is greater than the surrounding gel, has an energy velocity that is fairly close to, but nonetheless significantly larger than, either the group or phase velocities $\left(v_{\mathrm{e}}>v_{\mathrm{gr}}\right.$ and $\left.v_{\mathrm{e}}>v_{\mathrm{ph}}\right)$ throughout the entire frequency range of the measurements [Fig. 11(a)]. Note that these experimental values of the energy velocity for the GaIn system are not influenced by droplet resonances, which occur only at frequencies beyond the accessible range in the diffusion coefficient measurements. By contrast, as has been also reported previously [14], the energy velocity for the FC40 emulsion, for which the acoustic velocity of the droplets is smaller than the surrounding fluid, is substantially smaller than either the group or phase velocities $\left(v_{\mathrm{e}} \ll v_{\text {gr }}\right.$ and $\left.v_{\mathrm{e}}<v_{\text {ph }}\right)$ [Fig. 11(b)]. In this case, droplet resonances play a huge role in the behavior of the velocities, leading to large dispersion in the effective medium and a correspondingly large increase in the group velocity near the resonances, whereas the transport velocity of the multiply scattered waves $v_{\mathrm{e}}$ is significantly slowed down for the same 
frequencies [Fig. 11(b)]. Even between the resonances, the energy velocity for the FC40 emulsion is lower than any of the other velocities $\left(v_{\mathrm{ph}}, v_{\mathrm{gr}}, v_{0}\right)$.

To help understand these contrasting results for $v_{\mathrm{e}}$, we use a theory developed by van Tiggelen, van Albada, Lagendijk, and Tip $[18,19]$ for optical systems containing randomly distributed scatterers such as $\mathrm{TiO}_{2}$ particles. To emphasize the fundamental ideas, this theory was initially formulated for scalar waves, and is therefore especially applicable to our acoustic emulsion systems. In this theory, a generalized Boltzmann equation for the correlation function $\left\langle\psi\left(\mathbf{r}_{1}, t_{1}\right) \psi^{*}\left(\mathbf{r}_{2}, t_{2}\right)\right\rangle$ of the scalar wave function $\psi(\mathbf{r}, t)$ was solved in the Kubo limit for low concentrations of scatterers, when the self-energy and vertex function can be evaluated to leading order. The energy flux $J$ and energy density $U$ were expressed in terms of the Laplace transform with respect to time and the Fourier transform with respect to position of this correlation function, leading to a relation involving $J$ and $U$ that, once an equation of continuity was satisfied, yielded an expression for the energy velocity $v_{\mathrm{e}}$ of the following form:

$$
v_{\mathrm{e}}=\frac{v_{0}^{2} / v_{\mathrm{ph}}}{1+\delta},
$$

where the parameter $\delta$, rewritten in terms of the notation of this paper, is equal to

$$
\delta=2 \pi \eta v_{\mathrm{gr}}\left(\frac{v_{\mathrm{ph}}}{\omega} \frac{\partial \operatorname{Re} f(0)}{\partial \omega}+\int_{0}^{\pi} \sin (\theta)|f(\theta)|^{2} \frac{\partial \varphi_{s}(\theta)}{\partial \omega} d \theta\right) .
$$

The delay parameter $\delta$ accounts for the influence on $v_{\mathrm{e}}$ of the energy temporarily stored in the scattering inclusions during diffusive transport, and can lead to a very significant slowing down of the energy velocity when there are strong scattering resonances. As can be seen from Eq. (8), $\delta$ is the sum of two contributions, $\delta=\Delta_{1}+\Delta_{2}$, where

$$
\Delta_{1}=2 \pi \eta v_{\mathrm{gr}} \frac{v_{\mathrm{ph}}}{\omega} \frac{\partial \operatorname{Re} f(0)}{\partial \omega}
$$

and

$$
\Delta_{2}=2 \pi \eta v_{\mathrm{gr}} \int_{0}^{\pi} \sin (\theta)|f(\theta)|^{2} \frac{\partial \varphi_{s}(\theta)}{\partial \omega} d \theta .
$$

In order to interpret this expression for $v_{\mathrm{e}}$ [Eq. (7)], it is instructive to write the group velocity in terms of a group delay parameter $\Delta_{\mathrm{gr}}$. By determining the group velocity from $v_{\mathrm{gr}}=\partial \omega / \partial k$ using the ISA expression, Eq. (5), for the wave number, we obtain

$$
v_{\mathrm{gr}}=\frac{v_{0}^{2} / v_{\mathrm{ph}}}{1+\Delta_{\mathrm{gr}}}, \quad \text { with } \quad \Delta_{\mathrm{gr}}=\frac{v_{0}^{2}}{v_{\mathrm{ph}} v_{\mathrm{gr}}} \Delta_{1} .
$$

In the absence of resonances or when the resonances are weak, $v_{0}^{2} / v_{\mathrm{ph}} v_{\mathrm{gr}} \approx 1$ and we have $\Delta_{\mathrm{gr}} \approx \Delta_{1}$. The $\Delta_{1}$ term then corresponds to the group delay contribution to the energy velocity. The $\Delta_{2}$ term corresponds to the additional contribution [16] that accounts for the scattering delay given by the angular averaged phase shift $\partial \varphi_{s} / \partial \omega$ of the scattered wave in each scattering event. As predicted by van Tiggelen et al. [19], this scattering contribution $\Delta_{2}$ compensates the very large or even negative values of the group delay that may appear around resonances and ensures that the energy velocity

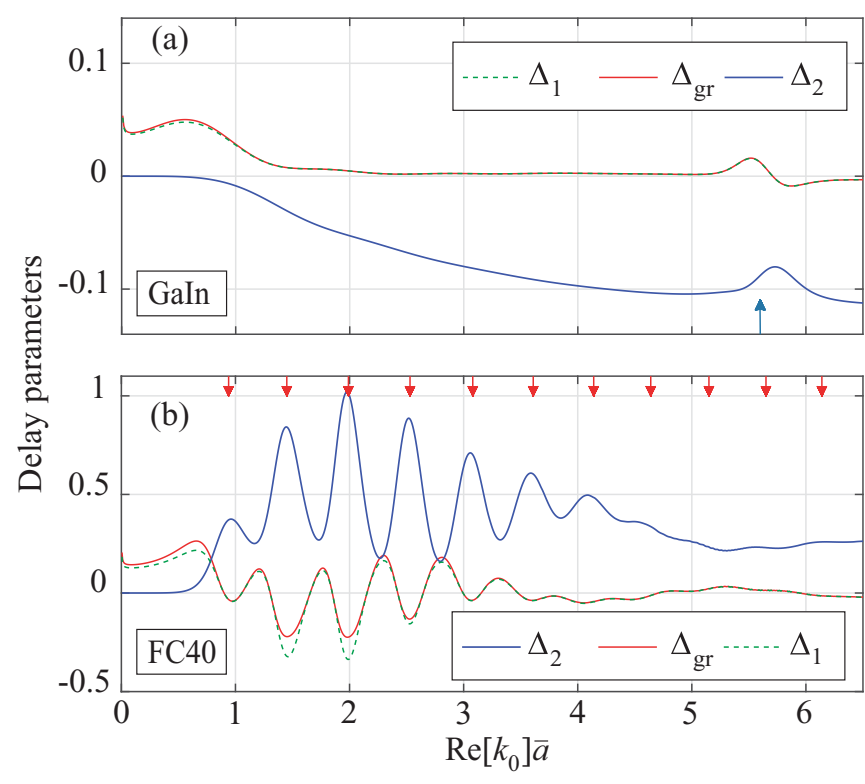

FIG. 12. Delay parameters $\Delta_{1}, \Delta_{2}$, and $\Delta_{\mathrm{gr}}$ calculated from scattering properties of a GaIn suspension with $\phi_{\mathrm{GaIn}}=16 \%$ and $\bar{a}=0.31 \mathrm{~mm}$ (a) and a FC40 emulsion with $\phi_{\mathrm{FC} 40}=5 \%$ and $\bar{a}=$ $0.17 \mathrm{~mm}$ (b). These results are presented for polydisperse populations of scatterers with $P_{\mathrm{GaIn}}=2 \%$ and $P_{\mathrm{FC} 40}=4 \%$. The black arrows represent the resonant frequencies of an isolated droplet. Note that the $y$-axis scale in (a) extends over a much smaller range of values than in (b).

properly accounts for the energy carried by the elastically scattered waves.

These three different delay parameters $\Delta_{1}, \Delta_{2}$, and $\Delta_{\mathrm{gr}}$ are plotted as a function of frequency in Fig. 12 for the two different samples. This calculation shows the close similarity between $\Delta_{1}$ and $\Delta_{\mathrm{gr}}$ at frequencies away from resonances (in particular for the GaIn suspension [Fig. 12(a)] for which dispersion effects are weak). Furthermore, in the case of fluorinated oil droplets [Fig. 12(b)], $\Delta_{2}$ and $\Delta_{\text {gr }}$ vary in the opposite way with frequency (when $\Delta_{2}$ increases with frequency $\Delta_{\mathrm{gr}}$ decreases with frequency, and vice versa), thereby explaining the large difference between $v_{\mathrm{e}}$ and $v_{\mathrm{gr}}$ for this system. This effect is due to the low sound speed within the scatterers, which causes a large radiative delay of the energy that is temporarily stored within the droplet. This difference between $v_{\mathrm{gr}}$ and $v_{\mathrm{e}}$ was predicted in optics $[18,19]$ but direct experimental confirmation was lacking due to the difficulty in measuring simultaneously the group and energy velocities of light in heterogeneous media.

Previous experiments using ultrasound [6,7] have also demonstrated the impact of the scattering delay on the energy velocity, but in these cases both the energy and group velocities were reduced and did not exhibit such opposite behavior as is shown in Fig. 11(b) [34]. These observations were due to the different character of the resonances in these samples containing hard solid scatterers with high sound speeds relative to the surrounding medium (the usual case in ultrasonic experiments). For example, in the case of concentrated suspensions of glass beads in a fluid, the dominant resonance at low frequencies may be described as a tortuosity resonance [9] 
that causes both the energy and group velocities to be reduced. Their results were interpreted using a model [6] motivated by their experimental findings for energy transport by wave pulses: the energy velocity was determined from the group velocity by accounting for the additional scattering delay that the multiply scattered waves experience as they are scattered through all possible scattering angles. This additional delay was expressed directly in terms of the intensity-weighted angular average $\Delta t_{\text {ave }}$ of the scattering time delay $\partial \varphi_{s}(\theta) / \partial \omega$,

$$
\Delta t_{\mathrm{ave}}=\frac{\int d \theta \sin \theta|f(\theta)|^{2} \frac{\partial \varphi_{s}}{\partial \omega}}{\int d \theta \sin \theta|f(\theta)|^{2}},
$$

where the normalization factor in the denominator is proportional to the cross section, and hence equal to $1 /\left(2 \pi \eta \ell_{\mathrm{s}}\right)$. Thus $\Delta t_{\mathrm{ave}}=\Delta_{2}\left(\ell_{\mathrm{s}} / v_{\mathrm{gr}}\right)$. If we start from Eq. (7) and write $v_{\mathrm{e}}$ explicitly in terms of $\Delta_{\mathrm{gr}}$ and $\Delta t_{\mathrm{ave}}$, we obtain

$$
\begin{aligned}
\frac{1}{v_{\mathrm{e}}} & =\frac{v_{\mathrm{ph}}}{v_{0}^{2}}\left(1+\Delta_{1}+\Delta_{2}\right) \\
& =\frac{v_{\mathrm{ph}}}{v_{0}^{2}}\left[1+\frac{v_{\mathrm{ph}} v_{\mathrm{gr}}}{v_{0}^{2}} \Delta_{\mathrm{gr}}\right]+\frac{v_{\mathrm{ph}} v_{\mathrm{gr}}}{v_{0}^{2}} \frac{\Delta t_{\mathrm{ave}}}{\ell_{\mathrm{s}}} .
\end{aligned}
$$

When the dispersion is weak so that $v_{0}^{2} / v_{\mathrm{ph}} v_{\mathrm{gr}} \approx 1$, Eq. (13) becomes

$$
\frac{1}{v_{\mathrm{e}}} \approx \frac{1}{v_{\mathrm{gr}}}+\frac{\Delta t_{\mathrm{ave}}}{\ell_{\mathrm{s}}},
$$

which is the approximate expression for $v_{\mathrm{e}}$ used successfully to interpret the data in Refs. [6] and [7]. The interpretation of this expression is more straightforward than Eq. (7), since Eq. (14) shows very clearly that $v_{\mathrm{e}}$ is determined by the sum of a group delay and a multiple scattering delay. As shown in Fig. 11, this simple expression gives predictions in excellent agreement with Eq. (7) for the GaIn suspension over the entire frequency range, but slightly underestimates the minima in $v_{\mathrm{e}}$ near the sharp resonances of the FC40 emulsion.

In the case of the GaIn suspension, the calculation of the delay parameters shows the reason for the low dispersion in the sample, with $\Delta_{\text {gr }}$ nearly equal to $\Delta_{1}$ and both delay parameters very close to zero over most of the frequency range. While the magnitude of the scattering delay $\Delta_{2}$ is also small, the most striking feature is that $\Delta_{2}$ is negative over the entire frequency range investigated: the presence of particles with a fast acoustic velocity relative to the surrounding medium induces an advance of the scattered waves and $v_{\mathrm{e}}>$ $v_{\mathrm{gr}}$. For light scattering experiments with dielectric particles in vacuum, even in the simplest case of $v_{\mathrm{gr}} \approx c^{2} / v_{\mathrm{ph}}$ (with $c$ being the speed of light in vacuum), this kind of observation is impossible since the inequality $v_{\mathrm{e}}>v_{\mathrm{gr}}$ would lead to $v_{\mathrm{e}}>c$. In acoustics, however, where the wave speed $v_{0}$ of the surrounding medium is not an absolute quantity and is many orders of magnitude less than $c$, there is no such problem with $v_{\mathrm{e}}>v_{0}$. Furthermore, although quite weak, the GaIn droplet resonances still cause a slowing down of multiply scattered wave transport relative to the energy velocity at neighboring frequencies, since $\Delta_{2}$ exhibits peaks near the resonant frequencies.

As additional information that is relevant to understanding the mechanism underlying the large differences in energy

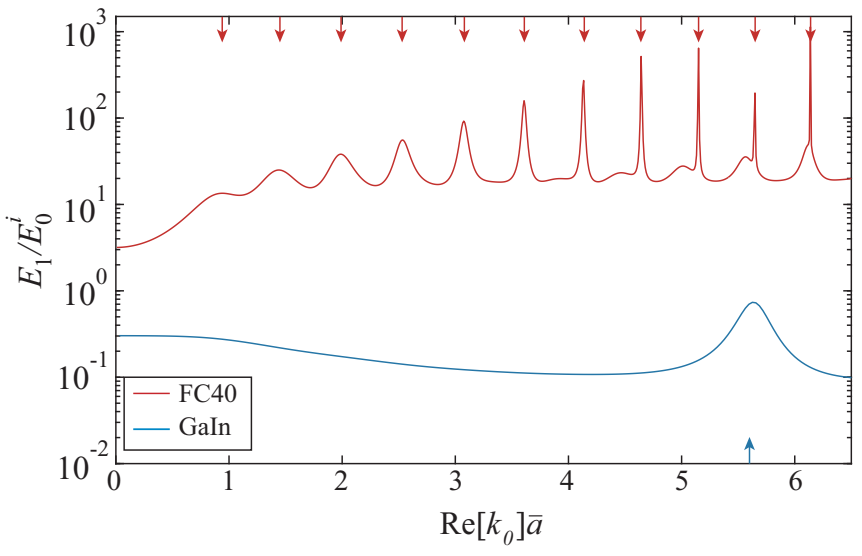

FIG. 13. Average acoustic energy divided by the droplet volume $E_{1}$ normalized by the energy density of the incident wave $E_{0}^{i}$. Red and blue arrows indicate the resonant frequencies for the FC40 and GaIn droplets, respectively.

velocities for the two emulsion systems, we show in Fig. 13 the calculated acoustic energy density within the droplets $E_{1}$ normalized by the incident acoustic energy density $E_{0}^{i}$. For an outline of the basic steps used in this calculation, please refer to the Appendix. As can be seen in Fig. 13, this calculation shows that the energy density within each scatterer is globally much higher for the FC40 droplets than for the GaIn droplets. In particular, the ratio $E_{1} / E_{0}^{i}$ is always much larger than 1 for the FC40 emulsion, whereas it is always much less than one for the GaIn emulsion over most of the frequency range. This difference in behavior is mostly due to the large difference in the acoustic velocities of FC40 and GaIn: the high velocity of GaIn relative to the matrix impedes the incident energy from entering and then being confined within the droplets, whereas the reverse is true for FC40 [35]. Furthermore, near the resonant frequencies of the droplets, peaks in the ratio $E_{1} / E_{0}^{i}$ are visible for both systems, and these peaks are especially large and narrow for the FC40 droplets. This identifies the mechanism for the scattering delays that cause the dips in energy velocity near these frequencies, confirming that the resonances lead to trapping of the waves within the scatterers.

\section{CONCLUSION}

Ultrasonic experiments $[2,5-7,14]$ can provide direct observation of the transmitted wave field through strongly scattering media with excellent temporal and spatial resolution, thereby enabling both the average wave and multiply scattered coda waves to be measured. Hence, as shown in this work, a complete set of wave scattering and transport parameters is accessible to experiment, unlike in optics where a similar set of measurements seems to be almost prohibitively challenging $[8,18,21,36,37]$. We have used this advantage of ultrasonic experiments to investigate wave transport in two contrasting model systems consisting of almost monodisperse emulsions, with either FC40 or GaIn droplets suspended in yield-stress fluids. The all-fluid character of these systems ensures that only scalar (acoustic) waves are involved, simplifying both the relevant theoretical models and the character of the droplet 
resonances, which follow a simple multipolar sequence. The very low intrinsic absorption in the fluids constituting these emulsion samples is another favorable characteristic, enabling the exploration of ultrasonic wave transport over a wide temporal range. In addition, emulsion samples with low droplet concentrations were fabricated so that models based on the independent scattering approximation or solutions of the Boltzmann transport equation in the low concentration limit would be applicable, and were found to accurately describe the experimental data.

This combination of experiments and theory has enabled us to show unambiguously how changing the relative refractive index of the scatterers from being greater than 1 to less than 1 impacts wave transport in the multiple scattering regime. While the influence of the relative index $n_{\text {rel }}$ on both the scattering and transport parameters is very significant, it is its effect on the energy velocity $v_{\mathrm{e}}$ that seems the most striking and unexpected. For the FC40 emulsion, with droplet index $n_{\text {rel }}=2.3$ (similar to $n$ for $\mathrm{TiO}_{2}$ particles in optics), we confirm that the resonances are strong and $v_{\mathrm{e}}$ is very substantially slowed down near these resonances by as much as a factor of 2 relative to the matrix velocity $v_{0}$, while the group velocity reaches very large values at these frequencies $\left(v_{\mathrm{gr}} \gg v_{0}\right)$. By contrast to this emulsion containing high refractive index droplets where $v_{\mathrm{e}} \ll v_{\mathrm{gr}}$, we find the opposite behavior in the GaIn emulsion $\left(v_{\mathrm{e}}>v_{\mathrm{gr}} \approx v_{0}\right)$, an effect that we have shown is due to the high sound speed in GaIn $\left(n_{\text {rel }}=0.56\right)$. The low relative refractive index of GaIn in this all-fluid scalar wave system results in a shift to higher frequencies of the multipolar resonances, which are also quite weak by comparison with FC40. Consequently, there is an appreciable range of frequencies over which there is strong multiple scattering but no droplet resonances occur, and it is at these frequencies where the energy velocity is most clearly seen, both experimentally and theoretically, to be larger that any of the other velocities (albeit by only 10 to 20\%). This result was not anticipated, based on previous work on low index solid inclusions in a fluid matrix [6,7]. The mechanism behind this contrasting behavior of the energy velocity in these two emulsion systems is elucidated by our calculations of the energy density in a droplet relative to the energy density of the incident wave; these calculations show that for high index droplets, $E_{1} / E_{0}^{i} \gg 1$, pointing to resonant trapping of energy inside the droplets as being responsible for the low energy velocity, while for low index droplets, $E_{1} / E_{0}^{i}<1$ in the strong scattering regime, and the waves are excluded from the droplets rather than being trapped inside.

The excellent control of sample properties that are feasible through soft-matter techniques [38] suggests that it will be interesting to explore other scalar wave systems in acoustics by further tuning the sound speed contrast between soft scatterers and the surrounding fluid medium, the strength of the resonances, and the concentration of the scattering inclusions. In this way, it may be possible to study wave transport in model systems with even stronger scattering, and maybe even to observe complex phenomena such as Anderson localization. The experimental study of Anderson localization for scalar classical waves in three dimensions would be a new experimental avenue of exploration that complements previous work on the Anderson localization of elastic waves in mesoglasses [39-43], and would avoid the complexities that challenge observations of Anderson localization of light in three dimensions due to its vector nature $[44,45]$.

\section{ACKNOWLEDGMENTS}

This work was supported by the LabEx AMADEus (ANR10-LABX-42) within IdEx Bordeaux (ANR-10-IDEX-0302), i.e., the Investissements d'Avenir programme of the French government managed by the Agence Nationale de la Recherche, and the Natural Sciences and Engineering Research Council of Canada's Discovery Grant Program (RGPIN-2016-06042).

\section{APPENDIX: SCATTERING OF AN ACOUSTIC PLANE WAVE BY A LIQUID SPHERE}

\section{Pressure field expressions}

We consider a homogeneous liquid sphere of radius $a$ (with density $\rho_{1}$ and sound speed $\left.v_{1}\right)$ immersed in another fluid ( $\rho_{0}$, $v_{0}$ ). When a plane wave is incident on the sphere, the aim of the calculation is to find expressions for the pressure field amplitudes $A_{n}$ and $B_{n}$ of the waves that are scattered away from the sphere and refracted inside the sphere, respectively. Because of the symmetry of the problem, we use a spherical polar coordinate system $\left(\vec{e}_{r}, \overrightarrow{e_{\theta}}, \overrightarrow{e_{\phi}}\right)$ with its origin at the center of the sphere. Under these conditions, the incident plane wave [with wave vector $\vec{k}_{0}=\left(\omega / v_{0}\right) \vec{e}_{z}$ ] is expressed as

$$
\psi_{0}^{i}(r, \theta, t)=\sum_{n=0}^{\infty} i^{n}(2 n+1) j_{n}\left(k_{0} r\right) P_{n}(\cos \theta) e^{-i \omega t},
$$

where $j_{n}$ are spherical Bessel functions of order $n$ and $P_{n}$ are the Legendre polynomials. Under the same conditions, the scattered $\psi_{0}^{s}$ and refracted $\psi_{1}$ fields are expressed as

$$
\psi_{0}^{s}(r, \theta, t)=\sum_{n=0}^{\infty} i^{n}(2 n+1) A_{n} h_{n}\left(k_{0} r\right) P_{n}(\cos \theta) e^{-i \omega t}
$$

and

$$
\psi_{1}(r, \theta, t)=\sum_{n=0}^{\infty} i^{n}(2 n+1) B_{n} j_{n}\left(k_{0} r\right) P_{n}(\cos \theta) e^{-i \omega t},
$$

where $h_{n}$ are spherical Hankel functions of the first kind.

The partial wave amplitudes $A_{n}$ and $B_{n}$ are determined by using continuity conditions of pressure $\psi_{m}$ and displacement $\left(\overrightarrow{u_{m}}=\vec{\nabla} \psi_{m} / \rho_{m} \omega^{2}\right)$ for the two regions $m=0$ and $m=1$ at the surface $r=a$ of the sphere. Thus we obtain

$$
A_{n}=-\frac{q j_{n}^{\prime}\left(k_{0} a\right) j_{n}\left(k_{1} a\right)-j_{n}\left(k_{0} a\right) j_{n}^{\prime}\left(k_{1} a\right)}{q h_{n}^{\prime}\left(k_{0} a\right) j_{n}\left(k_{1} a\right)-h_{n}\left(k_{0} a\right) j_{n}^{\prime}\left(k_{1} a\right)}
$$

and

$$
B_{n}=A_{n} \frac{h_{n}\left(k_{0} a\right)}{j_{n}\left(k_{1} a\right)}+\frac{j_{n}\left(k_{0} a\right)}{j_{n}\left(k_{1} a\right)},
$$

where $q=\frac{\rho_{1} k_{0}}{\rho_{0} k_{1}}$ and $^{\prime}$ denotes the derivative with respect to the parameters $k_{m} a$. The $A_{n}$ and $B_{n}$ coefficients are then functions of incident wave frequency $\omega$. From Eq. (A5), the resonant frequencies $\omega_{r}$ are identified from the peaks of the modulus of $B_{n},\left|B_{n}\right|$. For $\omega=\omega_{r}$, a given order $n$ corresponds to a 
particular symmetry of the scattered wave pattern (monopolar $n=0$ or dipolar $n=1$ modes, for example).

\section{Energy density calculation}

In terms of the pressure $\psi$ and displacement $\vec{u}$ fields, the acoustic energy density associated with each partial wave $n$ in region $m$ is written as follows:

$$
E_{m}^{n}=\frac{1}{2} \frac{\left(\psi_{m}^{n}\right)^{2}}{\rho_{m} v_{m}^{2}}+\frac{1}{2} \rho_{m} \omega^{2}\left|\overrightarrow{u_{m}}\right|^{2} .
$$

Since the energy density associated with the scattered wave $E_{0}^{s}$ decreases slowly with distance from the scatterer, only reaching zero at infinity, the exact numerical calculation of the ratio $E_{1} / E_{0}$ is not possible. Thus, for the calculations in Fig. 13, we only consider the energy density of the incident wave $E_{0}^{i}$ (which is constant in the region $r>0)$ and the energy density within the droplet $E_{1}$ (which varies in the limited region $r \leqslant a$ ), and characterize the relative energy density inside a droplet by the ratio $E_{1} / E_{0}^{i}$.
[1] P. Sheng, Introduction to Wave Scattering, Localization, and Mesoscopic Phenomena, 2nd ed. (Springer, Heidelberg, 2006).

[2] J. H. Page, P. Sheng, H. P. Schriemer, I. Jones, X. Jing, and D. A. Weitz, Science 271, 634 (1996).

[3] R. K. Snieder and J. A. Scales, Phys. Rev. E 58, 5668 (1998).

[4] Z. Q. Zhang, I. P. Jones, H. P. Schriemer, J. H. Page, D. A. Weitz, and P. Sheng, Phys. Rev. E 60, 4843 (1999).

[5] J. H. Page, H. P. Schriemer, A. E. Bailey, and D. A. Weitz, Phys. Rev. E 52, 3106 (1995).

[6] H. P. Schriemer, M. L. Cowan, J. H. Page, P. Sheng, Z. Liu, and D. A. Weitz, Phys. Rev. Lett. 79, 3166 (1997).

[7] N. Viard and A. Derode, J. Acoust. Soc. Am. 138, 134 (2015).

[8] P. M. Johnson, A. Imhof, B. P. J. Bret, J. G. Rivas, and A. Lagendijk, Phys. Rev. E 68, 016604 (2003).

[9] M. L. Cowan, K. Beaty, J. H. Page, Z. Liu, and P. Sheng, Phys. Rev. E 58, 6626 (1998).

[10] A. Derode, A. Tourin, and M. Fink, Phys. Rev. E 64, 036605 (2001).

[11] V. Leroy, A. Strybulevych, J. H. Page, and M. G. Scanlon, J. Acoust. Soc. Am. 123, 1931 (2008).

[12] M. L. Cowan, J. H. Page, and P. Sheng, Phys. Rev. B 84, 094305 (2011).

[13] T. Brunet, S. Raffy, B. Mascaro, J. Leng, R. Wunenburger, O. Mondain-Monval, O. Poncelet, and C. Aristégui, Appl. Phys. Lett. 101, 011913 (2012).

[14] B. Tallon, T. Brunet, and J. H. Page, Phys. Rev. Lett. 119, 164301 (2017).

[15] L. L. Foldy, Phys. Rev. 67, 107 (1945).

[16] A. Lagendijk and B. A. van Tiggelen, Phys. Rep. 270, 143 (1996).

[17] X. Jing, P. Sheng, and M. Zhou, Phys. Rev. A 46, 6513 (1992).

[18] M. P. van Albada, B. A. van Tiggelen, A. Lagendijk, and A. Tip, Phys. Rev. Lett. 66, 3132 (1991).

[19] B. A. van Tiggelen, A. Lagendijk, M. P. van Albada, and A. Tip, Phys. Rev. B 45, 12233 (1992).

[20] K. Busch, C. M. Soukoulis, and E. N. Economou, Phys. Rev. B 52, 10834 (1995).

[21] R. Sapienza, P. D. García, J. Bertolotti, M. D. Martín, A. Blanco, L. Viña, C. Lopez, and D. S. Wiersma, Phys. Rev. Lett. 99, 233902 (2007).

[22] P. D. García, R. Sapienza, J. Bertolotti, M. D. Martín, A. Blanco, A. Altube, L. Viña, D. S. Wiersma, and C. Lopez, Phys. Rev. A 78, 023823 (2008).

[23] The acoustic index is defined here as a "relative" index since no absolute quantity (such as light speed in vacuum) exists for acoustic waves. $n_{\text {rel }}=v_{0} / v_{1}$ is then the index of the scattering particle with sound speed $v_{1}$ relative to the surrounding medium with speed $v_{0}$, which in these experiments was usually similar to that of water.

[24] The yield stress is of the order of a few $\mathrm{Pa}$ [13], which is sufficiently high that sedimentation of droplets of our size and density is prohibited.

[25] B. Mascaro, T. Brunet, O. Poncelet, C. Aristégui, S. Raffy, O. Mondain-Monval, and J. Leng, J. Acoust. Soc. Am. 133, 1996 (2013).

[26] The ultrasonic absorption in this GaIn alloy was not measured, but is expected to be very small, as the absorption in liquid gallium has been reported to be exceptionally low $\left(1.6 \times 10^{-6} f^{2} \mathrm{~mm}^{-1}\right.$, with $f$ in $\left.\mathrm{MHz}\right)$ [J. L. Hunter and K. S. Hovan, J. Acoust. Soc. Am. 36, 1040 (1964)].

[27] A. S. Utada, E. Lorenceau, D. R. Link, P. D. Kaplan, H. A. Stone, and D. A. Weitz, Science 308, 537 (2005).

[28] M. D. Dickey, ACS Appl. Mater. Interfaces 6, 18369 (2014).

[29] R. Tadmouri, M. Romano, L. Guillemot, O. Mondain-Monval, R. Wunenburger, and J. Leng, Soft Matter 8, 10704 (2012).

[30] G. Lévêque, E. Rosenkrantz, and D. Laux, Meas. Sci. Technol. 18, 3458 (2007).

[31] H. Carslaw and J. Jaeger, Conduction of Heat in Solids, Oxford Science Publications (Clarendon Press, Oxford, 1959).

[32] J. J. Faran, J. Acoust. Soc. Am. 23, 405 (1951).

[33] A. Ishimaru, Wave Propagation and Scattering in Random Media (Academic Press, New York, 1978).

[34] Reference [7] does not contain an explicit comparison of energy and group velocities, but earlier work by Derode et al. on this system of steel rods in water [10] did show a significant slowing down of the group velocity near the lower of the two resonant frequencies for which a slowing down of the energy velocity was found in [7]. However, no dramatic change in the group delay was found in [10] near the second, higher frequency, resonance.

[35] To confirm that it is the velocity $\left(v_{1}\right)$ rather than the acoustic impedance $\left(\rho_{1} v_{1}\right)$ of the droplets that is mostly responsible for the energy density differences, we have repeated these calculations for two artificial droplet materials with the same velocities as FC40 and GaIn but densities chosen so that the acoustic impedances match the surrounding fluid. For the impedancematched "pseudo GaIn," the ratio increases by a factor of about 4 , but still remains less that 1 for $k_{0} a>1.5$ and is between 20 and 50 times less than the impedance-matched "pseudo FC40" at frequencies away from its resonances (on resonance the ratio is as much as 500 times smaller). Thus, even when there is no difference in impedance between the droplets and 
the surrounding medium, the low velocity droplet has a greatly enhanced energy density, while the high velocity droplet still has a small $E_{1} / E_{0}^{i}$ ratio $\lesssim 1$.

[36] T. Sperling, L. Schertel, M. Ackermann, G. J. Aubry, C. M. Aegerter, and G. Maret, New J. Phys. 18, 013039 (2016).

[37] G. J. Aubry, L. Schertel, M. Chen, H. Weyer, C. M. Aegerter, S. Polarz, H. Cölfen, and G. Maret, Phys. Rev. A 96, 043871 (2017).

[38] S. Raffy, B. Mascaro, T. Brunet, O. Mondain-Monval, and J. Leng, Adv. Mater. 28, 1760 (2016).

[39] H. Hu, A. Strybulevych, J. H. Page, S. E. Skipetrov, and B. A. van Tiggelen, Nat. Phys. 4, 945 (2008).
[40] S. Faez, A. Strybulevych, J. H. Page, A. Lagendijk, and B. A. van Tiggelen, Phys. Rev. Lett. 103, 155703 (2009).

[41] W. K. Hildebrand, A. Strybulevych, S. E. Skipetrov, B. A. van Tiggelen, and J. H. Page, Phys. Rev. Lett. 112, 073902 (2014).

[42] A. Aubry, L. A. Cobus, S. E. Skipetrov, B. A. van Tiggelen, A. Derode, and J. H. Page, Phys. Rev. Lett. 112, 043903 (2014).

[43] L. A. Cobus, S. E. Skipetrov, A. Aubry, B. A. van Tiggelen, A. Derode, and J. H. Page, Phys. Rev. Lett. 116, 193901 (2016).

[44] S. E. Skipetrov and I. M. Sokolov, Phys. Rev. Lett. 112, 023905 (2014).

[45] S. E. Skipetrov and J. H. Page, New J. Phys. 18, 021001 (2016). 